\title{
A Comparative Study of Patient Controlled Epidural Analgesia Using Bupivacaine and Fentanyl v/s IV Fentanyl in Patients for Pain Management in Post Operative Patients with Abdominal Surgeries
}

\author{
Authors \\ Rita Upadhyay, Ashish Sethi, Mayank Chansoriya \\ Department of Anesthesiology, NSCB Medical College, Jabalpur \\ Corresponding Author \\ Rita Upadhyay \\ A-87, Samdariya Residency, Shatabdipuram, Vijay Nagar, \\ Jabalpur, India 482003 Mobile No. 9179137714
}

\begin{abstract}
Patient Controlled Analgesia is system used in postoperative pain relief which enables patients to self titrate analgesia to their desired pain relief.This study has been a prospective study where patients were randomly allocated into two groups (30 patients each).Group A-Patients given general anaesthesia and post operative intravenous fentanyl (IV group).Group B-Patients given general anaesthesia and epidural catheter placed where post operative pain was managed with patient controlled epidural analgesia using fentanyl and bupivacaine (PCEA group).All patients received verbal and written instructions on the use of PCEA after taking informed consent. In IV Group:-The first dose of fentanyl (50 mcg ) was given on first analgesic request and then iv fentanyl $50 \mathrm{mcg}$ repeated every 4 hrs post operatively. PCEA Group: Epidural analgesia provided by post operative administration of $0.125 \%$ bupivacaine and $5 \mathrm{mcg} / \mathrm{ml}$ fentanyl mixture using a PCEA pump programmed to deliver $1 \mathrm{ml}$ bolus with a lock out interval of 20 minute and a background infusion of $2 \mathrm{ml}$ per hour. The cumulative fentanyl dose recorded during the $24 \mathrm{hrs}$ of the study. Postoperatively, the vital parameters, pain score and the side-effects of fentanyl were recorded at regular intervals for 24 hours. The overall pain scores were significantly lower in the PCEA group when compared to the IV group. The current study showed that postoperative PCA techniques by the epidural is more effective when compared with the parenteral route.

Keywords:- Pain, Patient Controlled Analgesia, Patient Controlled Epidural Analgesia, Fentanyl, Bupivacaine, lock out interval.
\end{abstract}

\section{Introduction}

The Taxonomy Committee of International Association for study of pain (IASP) defines pain as "an unpleasant sensory and emotional experience associated with actual or potential tissue damage or described in terms of such damage ${ }^{1}$. Post Operative pain is considered a form of acute pain to surgical trauma with a combined constellation of severe unpleasant sensory, emotional and mental experience precipitated by surgical trauma and associated with autonomic, endocrine, metabolic, physiologic and behavioural responses. ${ }^{2}$ Pain is a predictable part of the postoperative experience. ${ }^{3-5}$ Many options are available for the treatment of 
postoperative pain, including systemic (i.e.opioid and non opioid) analgesics and regional (i.e., neuraxial and peripheral) analgesic techniques but postoperative pain is still poorly treated ${ }^{6-8}$. Patient Controlled Analgesia (PCA) is system used in postoperative pain relief which enables patients to self titrate analgesia to their desired pain relief and then to maintain it at that level. PCA has been shown to offer a number of advantages, including good analgesia, avoidance of fluctuations in analgesia, lower total analgesic dosage, and improved patient satisfaction. In late 1960 s, the PCA pump was introduced by Philip H Sechzer .It was described in $1971^{9}$. Patient controlled epidural analgesia (PCEA) is a related term describing the patient controlled administration of analgesic medicine in the epidural space, by way of intermittent boluses or infusion pumps. Despite the introduction of new analgesics, advances in pain relief are most likely to come from improvements in the delivery of existing drugs to the patient. In the last few decades there have been two major advances in this field. The first was the introduction of spinal and epidural narcotics delivered locally to the 'target' spinal pain pathways. ${ }^{6-8,10,11}$ The second was the introduction of patient-controlled, ondemand analgesic systems which allow patients to titrate the amount of analgesic they receive directly against the amount of pain they are feeling. ${ }^{12-13} \mathrm{It}$ was considered that an on-demand analgesic system could provide the opportunity for the study of the potency duration of a narcotic when given by two different routes; in particular, the epidural and intravenous routes.

There is a need of a study comparing IV analgesia using fentanyl versus PCEA using bupivacaine and fentanyl for their efficacy on post operative pain so that the result can be generalized and post operative morbidity and mortality be reduced.

\section{Aims and Objectives}

To evaluate the efficacy of patient controlled epidural opioid (fentanyl) analgesia versus conventional regimens (intravenous fentanyl) of as-needed opioid analgesia for postoperative pain relief and patient satisfaction.

To compare the amount of opioid consumption between the two groups.

$>$ To evaluate side effects including nausea, vomiting, respiratory depression and other effects of opioids between the two groups.

\section{Materials and Method}

Place: N.S.C.B Medical College and Hospital, Jabalpur

\section{Selection of Cases}

An informed written consent was taken from all the patients in 2 groups after approval of institution's ethics committee. Patients of ASA class I and II of either sexes aged between 18 to 60 years scheduled for various abdominal surgeries in routine hours. A detailed history, thorough physical examination, routine investigations and any special investigation if required were done for the study.

\section{Criteria for exclusion}

* Patients with contraindications to epidural anaesthesia

* Patients with history of drug addiction, mental retardation and history of seizures.

* Patients with known hypersensitivity to local anaesthetic and study drugs.

* Patients with extreme malnutrition or cerebrovascular insufficiency.

* Patients with hypotension, and hemodynamic disturbances during surgery.

* Patients who have not signed the informed consent form.

\section{Design of Study}

A prospective study. Patients were randomly allocated into two groups (30 patients each).

Group A- Patients given general anaesthesia and post operative intravenous fentanyl (IV group).

Group B- Patients given general anaesthesia and epidural catheter placed where post operative pain was managed with patient controlled epidural analgesia using fentanyl and bupivacaine (PCEA group). 


\section{Method}

All patients received verbal and written instructions on the use of PCEA and to balance analgesia against sedation after taking informed consent the day before surgery. In OT, all the routine monitoring devices connected and 18G lines secured. A lumbar epidural catheter was placed at L3-L4 or L4-L5 level in group B patients only and confirmed with a test dose ( $3 \mathrm{ml}$ mixture of $2 \%$ Lignocaine with 1 in 50,000 dilution of Adrenaline). No drug was given via epidural catheter throughout the intraoperative period. After preoxygenation, IV Midazolam and IV Glycopyrrolate $0.4 \mathrm{mg}$ was given. IV fentanyl 2 microgram $/ \mathrm{kg}$ was given to blunt response of laryngoscopy. GA induced with IV Propofol 2-2.5 $\mathrm{mg} / \mathrm{kg}$. Intubation was facilitated using IV SCh 1.5$2 \mathrm{mg} / \mathrm{kg}$. The patients were mechanically ventilated by IPPV and anaesthesia was maintained with Isoflurane, N20 and O2. Vecuronium was used for muscle relaxation. Just 30 minutes before reversal, $100 \mathrm{ml}$ of IV paracetamol infusion was also given to all the patients in both the groups. At the end of the procedure, residual muscles relaxation was reversed using neostigmine $0.04 \quad-0.08 \quad \mathrm{mg} / \mathrm{kg}$ and glycopyrrolate $0.06 \mathrm{mg} / \mathrm{kg}$. Tracheal extubation performed with appropriate extubation criteria. After extubation patients were shifted to post anaesthesia care unit (PACU) and were given routine care with administration of oxygen by facemask and vital monitoring. On being fully awake post surgery, the use of the PCA device and VAS were explained again. In the PACU, patient received either fentanyl through intravenous route or through epidural route using PCA pump according to assigned group.

IV Group: The first dose of fentanyl (50 mcg ) was given on first analgesic request and then iv fentanyl $50 \mathrm{mcg}$ repeated every $4 \mathrm{hrs}$ post operatively. They received extra doses of iv fentanyl $25 \mathrm{mcg}$ when they experienced pain in between the 4 hourly dose. But this extra dose was given only once in between the 4 hourly interval.

PCEA Group: Epidural analgesia provided by post operative administration of $0.125 \%$ bupivacaine and $5 \mathrm{mcg} / \mathrm{ml}$ fentanyl mixture using a PCEA pump programmed to deliver $1 \mathrm{ml}$ bolus with a lock out interval of 20 minute and a background infusion of $2 \mathrm{ml}$ per hour. The cumulative fentanyl dose recorded during the $24 \mathrm{hrs}$ of the study. Postoperatively, the vital parameters, pain score and the side-effects of fentanyl were recorded at regular intervals for 24 hours. Patients were assessed by the anaesthesiologists on an hourly basis for the first 4 hrs after surgery, every 2 hours for the next 8 hours and then every $4 \mathrm{hrs}$ for the next 12 hours. Patients were assessed for pain, sedation, pulse rate, blood pressure, respiratory rate, oxygen saturation and side effects such as nausea, vomiting, pruritus, hypotension, respiratory depression etc.

\section{Assessment of post operative pain}

Postoperatively, the pain score was recorded by using VAS between 0 to 10 (0- no pain, 10-most severe pain),initially every $1 \mathrm{hr}$ for $2 \mathrm{hrs}$,then every $2 \mathrm{hr}$ for next $8 \mathrm{hrs}$ and then after every $4 \mathrm{hrs}$ till 24 hrs. IV paracetamol given as rescue analgesic whenever VAS $>4$.

\section{Consumption of fentanyl}

The cumulative fentanyl dose was recorded during the 24 hrs of the study.

\section{Assessment of side effects}

Patients were assessed for pain, sedation, pulse rate, blood pressure, respiratory rate, oxygen saturation and side effects such as nausea, vomiting, pruritus, hypotension, respiratory depression etc.

$>$ Intensity of sedation was evaluated by 5 point scaling. (Ramsay scale: 1. anxious, restless or both; 2.cooperative, orientated and tranquil; 3. responding to commands; 4. brisk response to stimulus; 5. sluggish response to stimulus; 6. no response to stimulus).

Hypotension is defined as a drop in systolic blood pressure of more than $20 \%$ of preoperative value or less than $90 \mathrm{mmHg}$ during the study period .

Respiratory depression is defined as respiratory rate of less than 10/min 


\section{Results}

The patients' demographics were similar between the PCEA and IV groups.

The total fentanyl requirement for adequate pain relief in the first 24 hours was less in the PCEA group as compared to the IV group.

The overall pain scores were significantly lower in the PCEA group when compared to the IV group.

At different intervals, the pain scores were lower in the PCEA group than the IV group.

The pain scores were lower in the later hours of the postoperative period in both the groups.

However, the decline in pain scores over time was greater in the PCEA group than the IV group .

No major fentanyl-related complications were noted. None of the patients in either group was unduly sedated or had respiratory depression.

\section{Nausea}

\begin{tabular}{|c|c|c|c|}
\hline Nausea & IV & PCEA & Total \\
\hline \multirow{2}{*}{ PRESENT } & 15 & 6 & 21 \\
\cline { 2 - 4 } & $50.00 \%$ & $20.00 \%$ & $35.00 \%$ \\
\hline \multirow{2}{*}{ Absent } & 15 & 24 & 39 \\
\cline { 2 - 4 } & $50.00 \%$ & $80.00 \%$ & $65.00 \%$ \\
\hline \multirow{2}{*}{ Total } & 30 & 30 & 60 \\
\cline { 2 - 4 } & $100.00 \%$ & $100.00 \%$ & $100.00 \%$ \\
\hline
\end{tabular}

$\mathrm{P}=0.015$, Significantly more nausea in IV group [ Chi square test]

\section{Visual Analogue Scale}

\begin{tabular}{|c|c|c|c|c|c|}
\hline \multirow{2}{*}{$\begin{array}{c}\text { VAS } \\
\text { Score }\end{array}$} & \multicolumn{2}{|c|}{ IV } & \multicolumn{2}{c|}{ PCEA } & \multirow{2}{*}{ p value } \\
\cline { 2 - 5 } & Mean & \pm SD & Mean & 土 SD & \\
\hline 0 HR & 2 & 0 & 2 & 0 & 1 \\
\hline 1 HR & 2.2 & 0.41 & 1.9 & 0.31 & 0.002 \\
\hline 2 HR & 2.6 & 0.5 & 1.4 & 0.5 & $<0.001$ \\
\hline 4HR & 3.5 & 0.68 & 1.3 & 0.47 & $<0.001$ \\
\hline 6 HR & 3 & 0.79 & 2.1 & 0.96 & $<0.001$ \\
\hline 8 HR & 2.2 & 0.41 & 2.5 & 0.51 & 0.014 \\
\hline 12 HR & 2.5 & 0.68 & 2.2 & 0.41 & 0.043 \\
\hline 16HR & 2.4 & 0.67 & 1.7 & 0.47 & $<0.001$ \\
\hline 20 HR & 2.2 & 0.61 & 1 & 0 & $<0.001$ \\
\hline 24 HR & 1.9 & 0.31 & 1 & 0 & $<0.001$ \\
& & & & & \\
\hline
\end{tabular}

Mean VAS score which was equal at 0 hours in both groups was significantly lower in PCEA group at all the recorded time intervals. $\mathrm{p}<0.05$
Vomiting

\begin{tabular}{|c|c|c|c|}
\hline Vomiting & IV & PCEA & Total \\
\hline \multirow{2}{*}{ Present } & 6 & 6 & 12 \\
\cline { 2 - 4 } & $20.00 \%$ & $20.00 \%$ & $20.00 \%$ \\
\hline \multirow{2}{*}{ Absent } & 24 & 24 & 48 \\
\cline { 2 - 4 } & $80.00 \%$ & $80.00 \%$ & $80.00 \%$ \\
\hline \multirow{2}{*}{ Total } & 30 & 30 & 60 \\
\cline { 2 - 4 } & $100.00 \%$ & $100.00 \%$ & $100.00 \%$ \\
\hline
\end{tabular}

$\mathrm{P}=1.00$, No significant differenc

Mean Fentanyl Consumption At 24 Hours

\begin{tabular}{|c|c|c|c|c|}
\hline Groups & $\begin{array}{c}\text { No of } \\
\text { patients }\end{array}$ & $\begin{array}{c}\text { Mean } \\
\text { fentanyl } \\
\text { dose }\end{array}$ & $\begin{array}{c} \pm \text { Std. } \\
\text { Deviation }\end{array}$ & $\begin{array}{c}\text { Std. Error } \\
\text { Mean }\end{array}$ \\
\hline IV & 30 & 402.5 & 17.8 & 3.2 \\
\hline PCEA & 30 & 243.5 & 3.3 & 0.6 \\
\hline & & & & \\
\hline
\end{tabular}

$\mathrm{P}<0.001$, Significantly more fentanyl required in IV group.

\section{Discussion}

It is challenging to relieve postoperative pain after major surgery without severe undesirable side effects. The nature of pain itself is subjective. Patient's response to analgesics is also variable and the efficacies of post-operative pain relief methods are neither uniform nor sufficient. Recent advances in the treatment of pain are the use of PCA. ${ }^{14,15}$ This can be used either intravenously (IVPCA) or epidurally (PCEA). Advantages of PCA over conventional pain management are that the therapy is individualized to the patient as per his subjective experience.

Epidural analgesia is nowadays a common and elegant technique in postoperative pain treatment. PCEA is not a routine method, however, and although similar in principle to IV PCA, it also involves the problems and risks of epidural puncture and catheterization. In our study, we observed that patient acceptability of PCA pump was good.

Due to lack of infrastructure, financial constrains and illiteracy among majority of patients it was challenging to explain the patients to use the device. 
However with full cooperation and coordination from medical and paramedical staff, and proper communication and counselling of relatives as well as patients, PCA pump could be used in our setup. It resulted in better pain relief, prevented many respiratory complications with earlier ambulation and recovery.

EA Welchew and D.P. Breen ${ }^{16}$, conducted a study comparing patient controlled on demand fentanyl delivered epidurally or intravenously. The study showed highly significant difference in fentanyl consumption between the two groups with the intravenous group demanding consistently more than twice as much as the epidural group.

In present study also the total fentanyl requirement for adequate pain relief in the first 24 hours was less in the PCEA group as compared to the IV group.

Cooper et al. ${ }^{17}$, showed that $0.125 \%$ bupivacaine with fentanyl $5 \mathrm{mcg} / \mathrm{ml}$ when delivered by PCA pump via epidural catheter decreased the requirement of one another.

In the similar study done by Behera et al. ${ }^{18}$ in 2008, comparison between IVPCA with morphine and PCEA with fentanyl and bupivacaine was done after thoracotomy procedures. They found that PCEA using fentanyl and bupivacaine provided better pain relief both at rest and during coughing and was associated with fewer side effects as compared to IV PCA using morphine.

In our study, variation in vitals were statistically significant specifically at 4 and 6 hours. Mean VAS score which was equal at 0 hours in both group was significantly lower in PCEA group at all the recorded time intervals. The overall pain scores were significantly lower in the PCEA group when compared to the IV group.

Neal Badner et al. ${ }^{19}$ in 1992 showed that $0.1 \%$ bupivacaine epidurally did not improve the quality of analgesia alone. But if it was combined with fentanyl, it definitely improved the quality of analgesia and decreased pain score. Epidural infusions of fentanyl, in a $10 \mathrm{mcg} / \mathrm{ml}$ concentration, combined with bupivacaine $0.1 \%$ were compared with epidural infusions of fentanyl alone for postoperative analgesia following abdominal or thoracic surgery. There were no detectable differences between the two groups in analgesia, no difference in postoperative pulmonary function or bowel function.

In present study the epidural route with a combination of local anaesthetics and a liposoluble opioid (fentanyl) resulted in better postoperative pain relief.

In the study by Claude Mann et al. ${ }^{20}$ pain relief was better at rest and after coughing in the PCEA group and the satisfaction scores were significantly greater in the PCEA group.

In the study conducted by Saito et al., ${ }^{21}$ the efficacy and safety of postoperative analgesia with continuous epidural infusion of either morphine or fentanyl in combination with bupivacaine were evaluated. They found that $18 \%$ patients developed significant hypotension in morphine bupivacaine group as compared to fentanyl bupivacaine group.

In the study conducted by Cooper et al. ${ }^{17}$ hypotension occurred in two patients in the fentanyl group, compared with eight in the bupivacaine group and 10 in the combined fentanyl and bupivacaine group.

In our study, not a single patient developed hypotension. In the study conducted by Mann et al. ${ }^{20}$ five episodes of postoperative hypotension occurred in the PCEA group versus none in the PCA group. The patients were treated by simple fluid loading.

Saito et al. ${ }^{21}$ in 1994 and Cooper et al. found no respiratory depression in their study. In both studies bupivacaine with fentanyl was used via the epidural route. Similar to the study conducted by Cooper et al., ${ }^{17}$ in our study no respiratory depression was observed in any of the patients of either group and all the patients were arousable.

Walder et al., ${ }^{22}$ in a meta-analysis, analyzed 32 trials in which morphine, pethidine (meperidine), piritramide, nalbuphine, or tramadol had been administered either by PCA or intramuscularly, intravenously, or subcutaneously. The evidence shows that, in the postoperative setting, opioid PCA, compared with conventional opioid treatment, improves analgesia and decreases the risk of 
pulmonary complications. In present study no major fentanyl-related complications were noted. None of the patients in either group were unduly sedated or had respiratory depression. Also there were no cases of hypotension in either group. The better analgesic effectiveness with respect to low incidence of side effects could probably explain the superiority of PCEA techniques. In addition, it is important to emphasize that the PCEA regimen was only partly patient controlled and offers the possibility of setting a background infusion. Background infusion improves analgesia achieved by PCEA without further side effects.

With epidural analgesia, the risk of orthostatic hypotension and motor blockade of the lower limbs during postoperative mobilization could counteract the benefit of the accelerated postoperative recovery. A self-adjustment by the patient probably explains in the current study the lack of significant hemodynamic instability and motor blockade and, in turn, does not interfere with the possibility of earlier ambulation.

Better post operative analgesia, decreased fentanyl consumption and less side effects may have occurred because we have included hemodynamically stable patients of ASA grade $1 \& 2$ which were posted for elective surgeries.

\section{Conclusion}

The current study showed that postoperative PCA techniques by the epidural is more effective when compared with the parenteral route. A background infusion using PCEA with a solution containing bupivacaine and fentanyl gave a better quality of analgesia, decreased the incidence of postoperative pain and had better acceptability, without any significant side effect.

\section{References}

1. Mersy H. Pain terms ; A list with definition and notes on usage. Recommended by the IASP subcommittee on Taxonomy. Pain. 1979; 6; 249-252.

2. Jorgen BD, Kehlet H; Post operative pain and its management. Wall and Melzacks
Textbook of pain. 5th ed. Elsevier Churchill Livingstone; Philadephia; 2006. p635.

3. Wightmanja K.; A prospective survey of the incidence of postoperative pulmonary complications. British Journal of Surgery 1968; 55 85-91.

4. Pfluge E, Bonicaj J.; Physiopathology and control of postoperative pain. Archives of Surgery 1977; 112: 773-81.

5. Spence AA.; Relieving acute pain. British Journal of Anaesthesia 1980; 522454.

6. Cohen FL. ;Post surgical pain relief: patients' status and nurses' medication choices. Pain 1980; 9: 265-74.

7. Marks RM, Sachar EJ. ;Undertreatment of medical inpatients with narcotic analgesics. Annals of Internal Medicine

8. Webs Of, Sriwatanakku, All Lozaj L, Weintraugm, Lasagnal ;Attitudes of patients, house staff and nurses towards postoperative analgesic care. Anaesthesia and Analgesia 1983; 62 7W.

9. Pearce, Jeremy ; "Philip H. Sehazer, 90, Expert of pain and how to ease it". New York Times, Retrieved 2010-11-22.

10. Wangj, K, Naussl A, Thomajes; Pain relief by intrathecally applied morphine in man. Anesthesiology 1979; 50 149-51.

11. Beharh, Olshwang, M Agorfa, Davidsojtn; Epidural morphine in treatment of pain. Lancet 1979; 1: 527-8.

12. Tamsena, Hartvicb, Dahlstrobm, Lindstromb, Holmdahmlm;Patient controlled analgesic therapy in the early postoperative period. Acta Anaesthesiology Scandinavica 1979; 23: 462-70.

13. Karanikolas M, Aretha D, Kiekkas $P$, Monantera G, Tsolakis I, Filos KS (October 2010). "Case report. Intravenous fentanyl patient-controlled analgesia for perioperative treatment of neuropathic/ischaemic pain in haemodialysis patients: a case series". J Clin Pharm Ther. 35(5): 603-8. doi:10.1111/j.1365-2710.2009.01114.x. PMID20831684. 
14. Cathy S. Jewell; Chambers, James Q.; Chearney, Lee Ann; Romaine, Deborah S.; Candace B. Levy (2007). The Facts on File encyclopedia of health and medicine. New York: Facts on File. ISBN0-8160-6063-0.

15. EA Welchew and D.P. Breen; Patient Controlled on demand epidural fentanyl Anaesthesia; 1991, vol 46, 438-441.

16. Cooper DW, Turner G; Patient-controlled extradural analgesia to compare bupivacaine, fentanyl and bupivacaine with fentanyl in the treatment of postoperative pain. $\mathrm{Br} \mathrm{J}$ Anaesth. 1993; 70: 503-7.

17. Behare BK, Puri GD, Ghai B.; Patient controlled epidural analgesia with fentanyl and bupivacaine provides better analgesia than Intravenous morphine patient controlled analgesia for early thoracotomy pain.J Postgrad Med. 2008,54:86-90.

18. Badner NH, Komar WE. Bupivacaine $0.1 \%$ does not improve post operative epidural fentanyl analgesia after abdominal or thoracic surgery. Can J Anaesth. 1992; 39 : 330-6.

19. Claude Mann et al; comparison of intravenous of epidural patient controlled analgesia in the elderly after major Abdominal Surgery; 2000, 92-433-41 Lippincott Williams \& Wilkins.

20. Saito, Y., Uchida, H., Kaneko, M., Nakatani, T. and Kosaka,Y.; Comparison of continuous epidural infusion of morphine/ bupivacaine with fentanyl/ bupivacaine for postoperative pain relief. Acta Anaesthesiol Scand, 1994; 3398-401.

21. B. Walder et al; efficacy and safety of patient controlled opiod analgesia for acute post operative pain Acta Anaestheisa and Scand, 2001, 45; 795-804.Ready B, Oden R Chadwick $\mathrm{H}$, et al. Development of ananesthesiology-based postoperative pain management service.Anesthesiology 1988; 68: $100-6$. 